\title{
Influence of the Dynamic Ergodic Divertor on the Density Limit in TEXTOR
}

\author{
Y. Liang, H. R. Koslowski, F. A. Kelly, M. Z. Tokar, X. Loozen, G. Bertschinger, W. Biel, K. H. Finken, M. W. Jakubowski, \\ A. Krämer-Flecken, O. Zimmermann, M. Lehnen, G. Sergienko, R. C. Wolf, and TEXTOR Team \\ Institut für Plasmaphysik, Forschungszentrum Jülich GmbH, Association FZJ-EURATOM, 52425, Jülich Germany
} (Received 19 November 2004; published 16 March 2005)

\begin{abstract}
A significant influence of the dynamic ergodic divertor (DED) on the density limit in TEXTOR has been found. In Ohmic discharges, where without DED detachment normally arises at the density limit, a MARFE (multifaceted asymmetric radiation from the edge) develops when the DED is operated in a static regime. The threshold of the MARFE onset in the neutral beam heated plasmas is increased by applying $1 \mathrm{kHz}$ ac DED at the high-field side. The theoretical predictions based on the parallel energy balance taking poloidal asymmetries into account agree well with the experimental observation.
\end{abstract}

DOI: 10.1103/PhysRevLett.94.105003

PACS numbers: 52.55.Fa, 52.40.Hf

Introduction. - In both tokamaks and stellerators with low impurity content and strong auxiliary heating, MARFE (multifaceted asymmetric radiation from the edge) formation has been observed to be the main characteristic of the density limit [1-3]. The MARFE is usually located at the high-field side (HFS) near the inner wall in limiter tokamaks. In divertor tokamaks, the MARFE usually begins near the $X$ point, but can also move to the inner wall. The development of MARFEs potentially causes plasma disruptions. The understanding of the physical mechanism is therefore of extreme importance for future thermonuclear reactors such as ITER (International Thermonuclear Experimental Reactor).

Over the years, results from both experimental observations and theoretical models show that the interaction between plasma, wall, and recycling neutrals of the working gas plays an important role in the thermal instabilities leading to MARFE formation [3-6]. In the tokamak TEXTOR, the density limit has been significantly extended with fresh boronization or siliconization [3] and exceeded by a factor of 1.7 the Greenwald-density limit, $n_{e}^{\mathrm{GW}}\left(\mathrm{m}^{-3}\right)=1 \times 10^{17}\left(\mathrm{kA}^{-1} \mathrm{~m}^{-1}\right) I_{p} /\left(\pi a^{2}\right)$, with the total plasma current $I_{p}$ and minor radius $a$ measured in kA and $\mathrm{m}$, respectively [7]. Complete suppression of the MARFE has been achieved by controlled displacement of the plasma column to the low-field side (LFS) [5]. In all these experiments, the local recycling and impurity release at the inner wall was reduced. Since the MARFE is strongly localized in the poloidal direction, it remains an open question whether the threshold value of the MARFE onset can be extended by making the distribution of recycling more homogeneous at the plasma edge.

Recently, the dynamic ergodic divertor (DED) has been installed at the high-field side of the tokamak TEXTOR (major radius $1.75 \mathrm{~m}$, minor radius of the circular plasma cross section $0.47 \mathrm{~m}$ ). The DED consists of 16 coils oriented parallel to the field lines on the magnetic surface with a safety factor equal to 3 . They extend symmetrically from the midplane in both directions up to a poloidal angle of $36^{\circ}$; two additional coils compensate for the imperfections at the feeder regions of the coils [8]. The resonant magnetic field perturbations caused by the DED introduce a complex three-dimensional stochastic magnetic field structure in the edge region, consisting of an ergodic zone formed by overlapping island chains and a laminar zone with short connection lengths of field lines to the wall. The DED has been used in $m / n=3 / 1$ and $12 / 4$ configurations, where $m$ and $n$ are the mode numbers of magnetic perturbations in poloidal and toroidal directions, respectively. The DED can be operated with static (dc) perturbation field or with the perturbation field rotating (ac) at frequencies of up to $10 \mathrm{kHz}$ in either the cocurrent or the countercurrent direction to the plasma current. Previously reported observations in TEXTOR with the $3 / 1$ configuration of the DED [9-11] include toroidal plasma rotation increased by the ac DED operation and the onset of the $m / n=2 / 1$ and $3 / 1$ tearing modes excited by the field perturbations. In this Letter we report the first results on the influence of the DED perturbation field on the density limit.

Experimental setup. - The investigation of the influence of the DED perturbation field on the density limit in TEXTOR has been done in Ohmic and neutral beam injection (NBI) heated plasmas with a plasma current of $300 \mathrm{kA}$ and a toroidal magnetic field of $2.25 \mathrm{~T}$, by using the $3 / 1$ configuration of the DED. The two-dimensional radiation profiles and the total radiated power are measured by using four bolometric cameras with metal foil resistor bolometers. Tomographic techniques have been applied to reconstruct the two-dimensional radiation profiles with a spatial resolution of $\sim 4 \mathrm{~cm}$ and a temporal resolution of $\sim 10 \mathrm{~ms}$ [12]. The plasma electron density, $n_{e}$, profiles are measured by a multichannel $\mathrm{HCN}$ laser interferometer [13].

Experimental results. - In Ohmic discharges without DED, no MARFE is observed and a poloidally symmetric radiation collapse occurs; i.e., the radiation power equals the total input power. A clear detachment appears when the electron density approaches $n_{e}^{\mathrm{GW}}$. However, when the dc 
DED is operated, a MARFE is observed at the HFS with a toroidally symmetric distribution. The MARFE appears at lower density when the DED coil current, $I_{\mathrm{DED}}$, is increased from 0.6 to $1.0 \mathrm{kA}$ and $3.75 \mathrm{kA}$, respectively, as shown in Fig. 1(a). For discharges with ac DED at $1 \mathrm{kHz}$ in both cocurrent and countercurrent directions no MARFE develops, and only a reduction of the density limit caused by plasma detachment is observed when $I_{\mathrm{DED}}$ is increased up to $1.4 \mathrm{kA}$. The corresponding period of the DED $X$-point motion over the divertor plate of $10^{-3} \mathrm{~s}$ is much longer than the time for the penetration of recycling neutrals into the plasma till their ionization, $\sim 10^{-5} \mathrm{~s}$, and of the plasma motion along on the magnetic surfaces with a sound velocity, $\sim 10^{-4} \mathrm{~s}$. Figures $1(\mathrm{~b})-1(\mathrm{~d})$ show the contours of line-integrated $H_{\alpha}$ line emission near the inner wall measured by a tangentially viewing charge-coupled
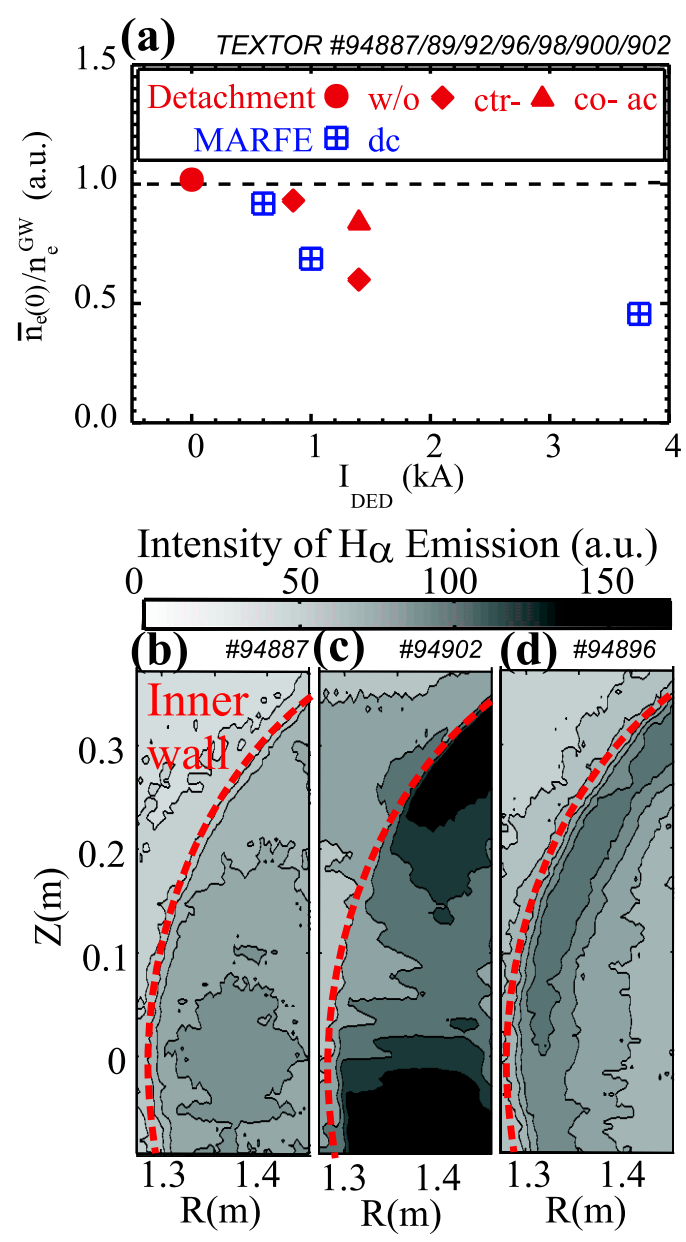

FIG. 1 (color online). (a) The central line-averaged electron density normalized to the Greenwald density $\left(n_{e}^{\mathrm{GW}}\right)$ as a function of the DED coil current $\left(I_{\mathrm{DED}}\right)$ measured in Ohmic only heated plasmas with dc DED (square), ac DED at $1 \mathrm{kHz}$ rotation in cocurrent (triangle) or countercurrent (diamond) directions, respectively. Comparison of the $H_{\alpha}$ emission at the HFS measured by a tangential CCD camera in the plasma: (b) without DED; (c) with dc DED $\left(I_{\mathrm{DED}}=3.75 \mathrm{kA}\right)$; (d) ac countercurrent $1 \mathrm{kHz}$ $\operatorname{DED}\left(I_{\mathrm{DED}}=0.85 \mathrm{kA}\right)$ at the time before the onset of a MARFE (c) or detachment (b),(d). device (CCD) camera at the time before the MARFE or detachment. The steep gradient at $R \sim 1.3 \mathrm{~m}$ is due to the shadow of the inner wall. The geometrical integration effect in front of the inner wall is relatively small, since the $H_{\alpha}$ line emission is mainly localized at the plasma edge. An increase of the recycling at the HFS, indicated by an increase of the intensity of the $H_{\alpha}$ line emission, has been observed for plasmas with both dc and ac DED. This is consistent with the reduction of the density limit observed in plasmas with DED. Furthermore, a difference in the particle recycling distribution pattern between dc and ac DED plasmas has been found. There are two strong recycling spots observed at the fixed strike zones in the plasmas with dc DED, while the recycling pattern appears more uniform at the inner wall when the DED is operated in the $1 \mathrm{kHz}$ ac regime. Similar features have also been observed in the two-dimensional radiation profiles measured as shown in Figs. 2(a) and 2(b). Two highly radiating regions localized in front of the DED at the HFS are observed in the plasmas with dc DED, while a poloidally symmetric radiation belt at the edge occurs in the plasmas with $1 \mathrm{kHz}$ ac DED. The slightly higher radiating spot at the LFS is the contribution from the ALT-II toroidal belt limiter of TEXTOR. The location of the strike zones observed by both the tangential CCD camera and the bolometric cameras agree with the theoretical prediction of the helical structure of the near field close to the inner wall [14].

A $m / n=2 / 1$ tearing mode generated by a $3 / 1$ configuration DED perturbation field has been observed in the plasmas when $I_{\mathrm{DED}}$ is larger than $0.7 \mathrm{kA}$ in the dc regime and $1.0 \mathrm{kA}$ in the $1 \mathrm{kHz}$ countercurrent ac regime. A $m / n=3 / 1$ tearing mode is observed with dc DED only when $I_{\mathrm{DED}}>2.3 \mathrm{kA}$ [11]. The frequency for both the $2 / 1$ and the $3 / 1$ modes are locked to the frequency of the external perturbation field; i.e., it is zero in the dc case. The difference in the reduction of the threshold of detachment between cocurrent and countercurrent ac DED with $I_{\mathrm{DED}}=1.4 \mathrm{kA}$ is mainly due to the onset of the $2 / 1$ tearing mode in the latter case.

In plasmas with strong auxiliary heating, the maximum electron density is usually limited by the MARFE onset. In

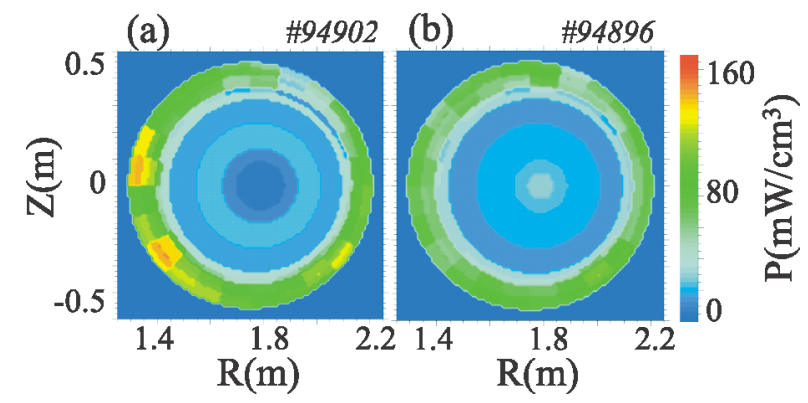

FIG. 2 (color). Comparison of two-dimensional radiation profiles measured by four bolometric cameras in plasmas with (a) dc $\operatorname{DED}\left(I_{\mathrm{DED}}=3.75 \mathrm{kA}\right)$ and $(\mathrm{b})$ ac countercurrent $1 \mathrm{kHz}$ DED $\left(I_{\mathrm{DED}}=0.85 \mathrm{kA}\right)$. 
experiments that investigate the influence of the DED divertor on impurity screening by puffing gas into the NBI heated plasmas from the HFS, MARFEs were also observed. Figure 3 compares the total radiated power for discharges without and with $1 \mathrm{kHz}$ cocurrent ac DED. In these experiments, neutral beams with a power of $1.3 \mathrm{MW}$ are injected into the plasma in the cocurrent direction from 0.8 to $5 \mathrm{~s}$. A small (20 mbarl) amount of $\mathrm{C}_{3} \mathrm{H}_{4^{-}}$gas has been puffed into the plasma from 2 to $4 \mathrm{~s}$ at the HFS. In the discharge without DED, the total radiation rapidly increases at $2.8 \mathrm{~s}$ by the generation of a MARFE. The corresponding central line-averaged electron density, $\bar{n}_{e}(0)$, reaches $3.7 \times 10^{19} \mathrm{~m}^{-3}\left(0.82\right.$ times $\left.n_{e}^{G W}\right)$. When the cocurrent ac DED at $1 \mathrm{kHz}$ with $I_{\mathrm{DED}}=2.55 \mathrm{kA}$ is applied, no MARFE is observed during the DED phase, even if $\bar{n}_{e}(0)$ increases to $3.9 \times 10^{19} \mathrm{~m}^{-3}$. However, a MARFE appears immediately after the DED is switched off. The results from measured $2 \mathrm{D}$ radiation profiles clearly show that the MARFE is generated from a highly radiating spot by the puffing of $\mathrm{C}_{3} \mathrm{H}_{4}$ and then moving up or down at the HFS. The disappearance of the MARFE in the later phase of the discharge is due to a shift of the plasma outward to the LFS to avoid a disruption caused by the MARFE onset.

Figure 4 shows the time evolution of $I_{p}, \bar{n}_{e}(0), P_{\mathrm{rad}}$, and the ratio of $H_{\alpha}$ to $H_{\gamma}$ intensities, $I_{H \alpha} / I_{H \gamma}$, for a discharge with $1 \mathrm{kHz}$ countercurrent ac DED in which the density was increased continuously. In this discharge, cocurrent NBI with a power of 1.3 MW has been injected into the plasma from 0.5 to $6.5 \mathrm{~s}$. The duration of the DED is from 0.7 to $5.9 \mathrm{~s}$ with a flattop of $0.85 \mathrm{kA}$, which is below the threshold for the onset of the $2 / 1$ tearing mode. Because of a limitation of the gas puffing system during these experiments, the ramp-up rate of $n_{e}$ did not exceed $0.6 \times$ $10^{19} \mathrm{~m}^{-3} \mathrm{~s}^{-1}$ even with the puffing valve completely opened. The electron density increased up to $5.0 \times$ $10^{19} \mathrm{~m}^{-3}$ (1.11 times $n_{e}^{\mathrm{GW}}$ ) without the observation of a MARFE during the DED phase. Just after switching off of the DED, a MARFE was formed at $t=5.95 \mathrm{~s}$. As a con-

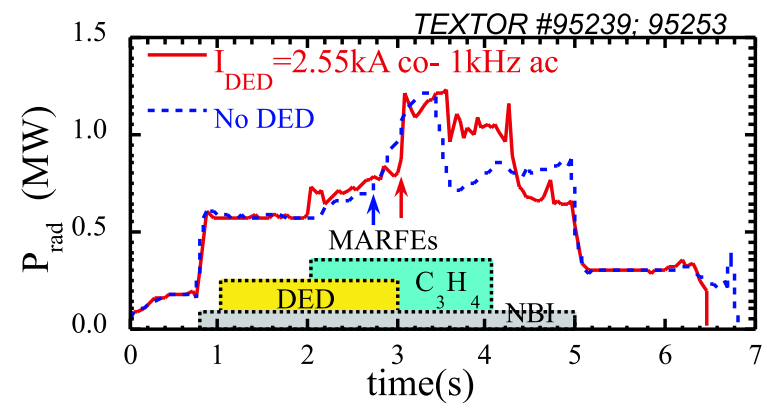

FIG. 3 (color online). Time evolution of the total radiated power, $P_{\text {rad }}$, in two NBI heated $(1.3 \mathrm{MW})$ discharges, without DED (dashed line) and with ac cocurrent $1 \mathrm{kHz}$ DED $\left(I_{\mathrm{DED}}=\right.$ $2.55 \mathrm{kA}$ ) (solid line). In both cases, a small (20 mbar 1) amount of $\mathrm{C}_{3} \mathrm{H}_{4}$ gas was puffed into the plasma at the HFS. sequence, a sudden increase of $P_{\text {rad }}$ and a reduction of $I_{H \alpha} / I_{H \gamma}$ has been observed.

Interpretation. - The observed variety of density limit scenarios and the influence of the DED can be interpreted by analyzing the stationary heat balance equation for the plasma edge:

$$
\Delta_{\text {edge }} \frac{\partial}{\partial l}\left(-K_{\|} \frac{\partial T}{\partial l}\right)=q_{\text {core }}-q_{\text {loss }} \text {, }
$$

where $\Delta_{\text {edge }}$ is the characteristic radial width of the edge region determined by the penetration depth of recycling neutrals, $K_{\|}$the heat conductivity along field lines in the direction $l, q_{\text {core }}$ the heat flux density from the plasma core, and $q_{\text {loss }}$ the energy losses at the edge including plasma conduction, convection, ionization, and excitation of recycling neutrals and impurities.

Because of the Shafranov shift $\Delta$ of the magnetic surfaces $q_{\text {core }}$ has a minimum at the HFS. In Ohmic plasmas with poloidal beta $\beta_{p}=0.32$ and internal inductance $\ell_{i}=$ 1.4 this asymmetry is, however, relatively small. The parameter $\Lambda=\beta_{p}+\ell_{i} / 2-1$, characterizing its level [15], is of 0.02 . The density limit under these conditions is caused by detachment and was explained in Ref. [16] as a result of a transition in edge turbulence to that driven by drift resistive ballooning modes. As a result, convective energy losses increase strongly, the plasma edge cools down and the impurity radiation grows. With strong enough NBI heating in TEXTOR $\left(\beta_{p}=0.6, \ell_{i}=1.1\right.$, and $\Lambda=0.15$ ), the Shafranov shift leads to a significant poloidal asymmetry of $q_{\text {core }}$. Therefore the thermal instability caused by the temperature dependence of the energy losses due to recycling [17] and impurity radiation [1] trigger a MARFE at the HFS before any transition in the edge turbulence can take place.

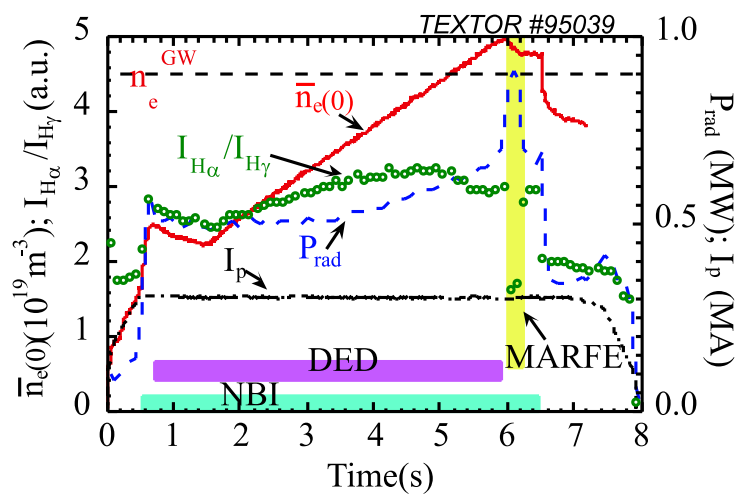

FIG. 4 (color online). Time evolution of the central lineaveraged electron density, $\bar{n}_{e}(0)$ (solid line), the ratio of $H_{\alpha}$ and $H_{\gamma}$ line emission, $I_{H \alpha} / I_{H \gamma}$ (open circles), total radiated power, $P_{\text {rad }}$ (dashed line), and plasma current, $I_{p}$ (dot-dashed line) for a discharge with NBI $\left(P_{\mathrm{NBI}}=1.3 \mathrm{MW}, t=0.5-6.5 \mathrm{~s}\right)$ heating and countercurrent ac $1 \mathrm{kHz} \operatorname{DED}\left(I_{\mathrm{DED}}=0.85 \mathrm{kA}\right.$; $t=0.7-5.9 \mathrm{~s})$. 


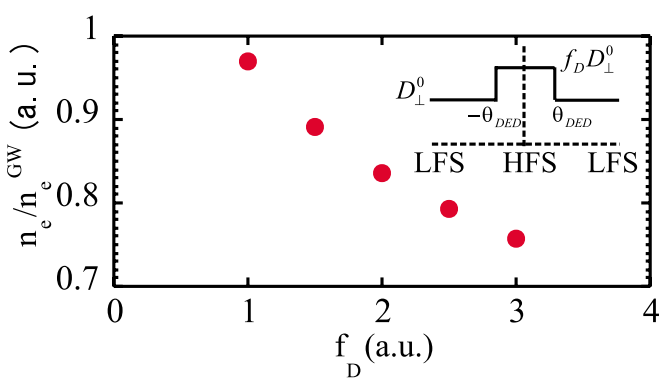

FIG. 5 (color online). Numerically found dependence of $\bar{n}_{e}(0) / n_{e}^{\mathrm{GW}}$ on $f_{D}$ for TEXTOR plasmas $\left(P_{\mathrm{NBI}}=1.3 \mathrm{MW}\right)$ [19].

With dc DED the recycling is stronger localized at the HFS and provides the asymmetry in $q_{\text {loss }}$. This enhances the asymmetry in $q_{\text {core }}$ induced by the Shafranov shift. As a result, in Ohmic discharges a MARFE develops instead of detachment and in discharges with NBI the density limit due to a MARFE is decreased. This is well reproduced in the modeling both of the linear instability threshold [18] and of the nonlinear MARFE evolution [19]. Figure 5 shows the calculated critical density at the MARFE onset as a function of the enhancement of the charged particle loss in front of DED coils (see the insertion in the figure) by the factor $f_{D}$ with respect to the case without DED.

In the cases with $1 \mathrm{kHz}$ ac DED, the smoothing of the recycling pattern at the HFS is due to a finite rate of the neutral desorption from the wall. If this rate is much smaller than that of the rotation frequency of the DED $X$ points, neutrals are released with a strong delay with respect to the moment when the ions were lost from the plasma. Therefore, the neutral influx is averaged over many periods of the DED current oscillations, and its spatial modulation is significantly reduced with respect to the case of fixed $X$ points (dc DED). In addition, the rotating near field lines of the DED can carry the charged particles preventing an accumulation of plasma in the region where the MARFE normally occurs and transporting heat to this position from surrounding warmer plasma regions. This explains why the density limit in Ohmic plasmas with the same DED current is higher in dc operation than in the ac regime. The nature of the limit is also changed from MARFE to detachment. The latter happens, however, at a density lower than without DED, since the recycling at the HFS is enhanced although smoothed. In NBI heated discharges, where the density limit is always due to MARFEs, it increases with ac DED with respect to the unperturbed case. Both the smoothing of recycling and poloidal flow of plasma particles with moving field lines can contribute to this effect. In order to unambiguously confirm the plasma edge flow induced by the rotating magnetic field, further developments of plasma edge diagnostics are needed on TEXTOR.

Recently, the density limit experiments with $12 / 4$ configuration of DED have also been performed on TEXTOR. No $m / n=2 / 1$ or $3 / 1$ tearing modes, caused by the DED, are observed even if $I_{\mathrm{DED}}$ is increased up to $10 \mathrm{kA}$ in dc and up to $5 \mathrm{kA}$ in the ac regime of operation. The preliminary results show that the density limit caused by the MARFEs in the NBI heated plasmas is reduced with dc DED $\left(I_{\mathrm{DED}}=10 \mathrm{kA}\right)$ and increased with $2 \mathrm{kHz}$ ac DED $\left(I_{\mathrm{DED}}=5 \mathrm{kA}\right)$. No difference in the density limit is observed in plasmas with a different rotation direction of DED, i.e., in cocurrent or countercurrent directions, which agrees with expectations based on the experimental results with the $3 / 1$ configuration of DED.

Conclusion. - It has been observed for the first time that the threshold of the MARFE onset in the NBI heated plasmas is increased by making a more homogeneous plasma recycling at the plasma edge by applying a $1 \mathrm{kHz}$ ac magnetic field perturbation at the HFS. The MARFE does not appear even if the $\bar{n}_{e}(0)$ reaches 1.11 times $n_{e}^{\mathrm{GW}}$ during the ac $1 \mathrm{kHz}$ DED phase. The experimental results show, in agreement with theoretical predictions, that the threshold of the MARFE onset strongly depends on both the level and the poloidal distribution of the recycling. This result may be of importance for the active control of MARFEs without changing the plasma configurations, i.e., the plasma position, in future fusion devices.

[1] B. Lipschultz et al., Nucl. Fusion 24, 977 (1984).

[2] U. Wenzel, K. McCormick, D. Hildebrandt, S. Klose, D. Naujoks, and H. Thomsen, Plasma Phys. Controlled Fusion 44, L57 (2002).

[3] J. Rapp et al., J. Nucl. Mater. 290-293, 1148 (2001).

[4] M.Z. Tokar and F. A. Kelly, Phys. Plasmas 10, 4378 (2003).

[5] P. de Vries et al., Phys. Rev. Lett. 80, 3519 (1998).

[6] J. Rapp et al., J. Nucl. Mater. 313-316, 524 (2003).

[7] M. Greenwald et al., Nucl. Fusion 28, 2199 (1988).

[8] K. H. Finken, Fusion Eng. Des. 37, 335 (1997).

[9] K.H. Finken et al., Physical Review Letters (to be published).

[10] H.R. Koslowski et al., in Proceedings of the 31st European Physical Society Conference on Plasma Physics, London, 2004 (European Physical Society, London, 2004), 28G, P1.124.

[11] Y. Liang et al., in Proceedings of the 31st European Physical Society Conference on Plasma Physics, London, 2004 (Ref. [10]), 28G, P1.126.

[12] J. Rapp, Ph.D. thesis, Berichte der Forschungszentrums Jülich, 1996, ISSN 0944-2952.

[13] H. R. Koslowski and H. Soltwisch, Fusion Eng. Des. 3435, 143 (1997).

[14] K. H. Finken et al., Plasma Phys. Controlled Fusion 46, B143 (2004).

[15] J. Wesson, Tokamaks (Oxford University Press, Inc., New York, 1997).

[16] M.Z. Tokar, Phys. Rev. Lett. 91, 095001 (2003).

[17] M.Z. Tokar et al., J. Nucl. Mater. 266-269, 958 (1999).

[18] F. A. Kelly and M.Z. Tokar, Contrib. Plasma Phys. 44, 176 (2004).

[19] F. A. Kelly, M.Z. Tokar, and Y. Liang, Bull. Am. Phys. Soc. 49, No. 8, 313 (2004). 